\title{
Comprehensive Nanofabrication by Correlating Crossbeam and ORION Nanofab
}

\author{
Ingo Schulmeyer ${ }^{1}$ \\ ${ }^{1}$ Carl Zeiss Microscopy GmbH, Carl-Zeiss-Straße 22, 73447 Oberkochen, Germany
}

Scanning electron microscopy (SEM) technologies provide only limited information about a certain sample. The gathered information is basically limited to the surface or areas near the surface of a sample. Combining SEM with a focused Gallium ion beam (Ga-FIB) opens a complete new application space for 3D Nanoanalysis and Nanofabrication. The analysis in three dimensions enables a better understanding of a samples electronic, magnetic, optical and mechanical properties. Many of these properties are defined by the structure in the size range below $100 \mathrm{~nm}$. To create superior materials and functional surfaces, fabrication in this size range becomes vital.

The Ga-FIB of a Crossbeam can be used to create structures with dimensions down to $30 \mathrm{~nm}$. By using a Helium or Neon ion beam, smaller structures with greater precision can be achieved. The He-beam is perfectly suited for creating smallest details like Nanopores or plasmonic devices between 3 and about $15 \mathrm{~nm}$. Neon ion fabrication bridges the Gap between Helium and Gallium and allows fast fabrication of e.g. nanowires (Figure 1). Both Microscopes can be equipped with a Gas injection system (GIS) to perform deposition of various materials or different etching processes.

With its unique Nanofabrication portfolio, ZEISS now introduces a workflow that allows the correlation of Fabrication and Analysis tasks between a Crossbeam FIB-SEM and an ORION Nanofab (Helium and Neon Ion beam instrument). This workflow allows to perform patterning and/or imaging operation in either microscope and navigate precisely to the same position.

The correlation of these technologies opens up a complete new application space in the materials research. We will demonstrate, how the Crossbeam provides a robust platform perfectly suited for common FIB-SEM applications like 3D-imaging and analytics, Automated Sample Preparation (Figure 2) or classical Ion Beam or Electron Beam Lithography (IBL, EBL). This allows a comprehensive characterization of the sample as well as the possibility to easily create special types of structures that will be post-processed by the ORION Nanofab. Larger functional surfaces can be created fast and easily using the high current Ga-ion beam.

After the initial analysis and pre-processing the specimen is transferred to the ORION Nanofab and aligned. The sample is recognized by the microscope making it easy to find all the regions of interest. The Helium and Neon beam are used to finalize the fabrication of the desired structures (Figure 3). More than that, Helium and Neon ions can be used for sub-nm imaging with unique surface sensitivity, high depth of field and the ability to easily image insulating samples without charging effects [1]. We demonstrate the technical realization of the workflow on different examples from the materials sciences and nanofabrication.

\section{References:}

[1] B. Ward, J.A. Notte, N.P. Economou, Helium Ion Microscopy, Photonics Spectra 41.8 (2007), p. $68-70$ 


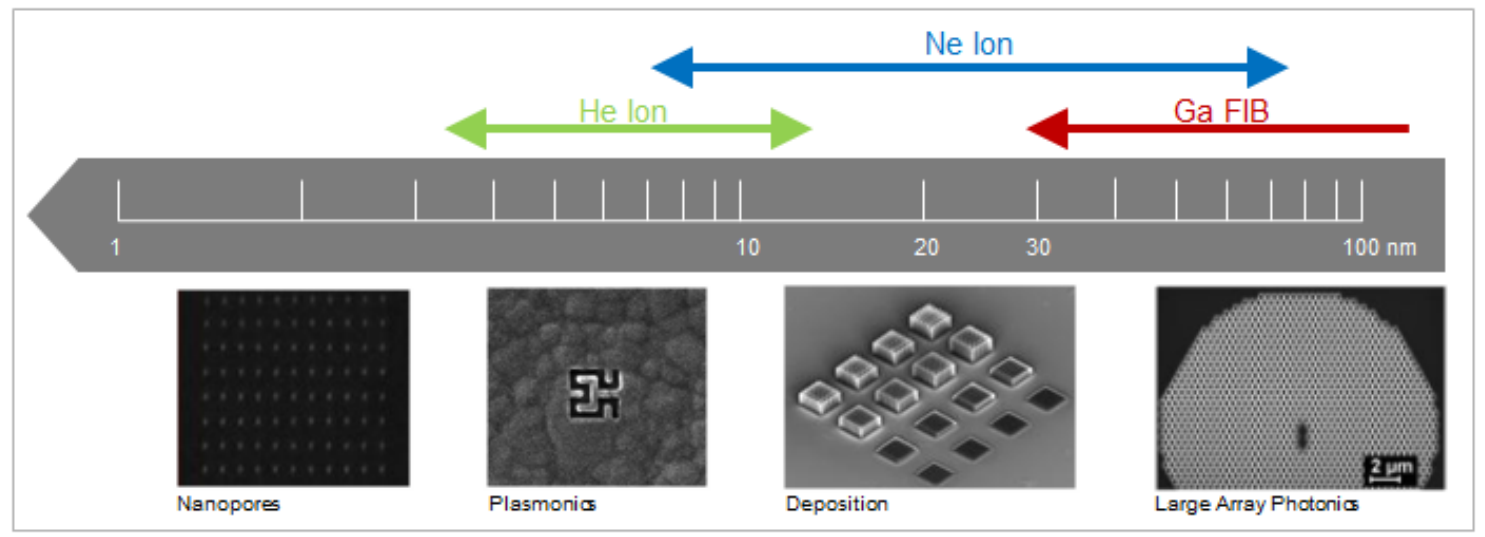

Figure 1. The combination of Helium, Neon and Gallium Focused Ion Beams allows Nanofabrication of features with several hundred nanometer down to only a few nanometer.
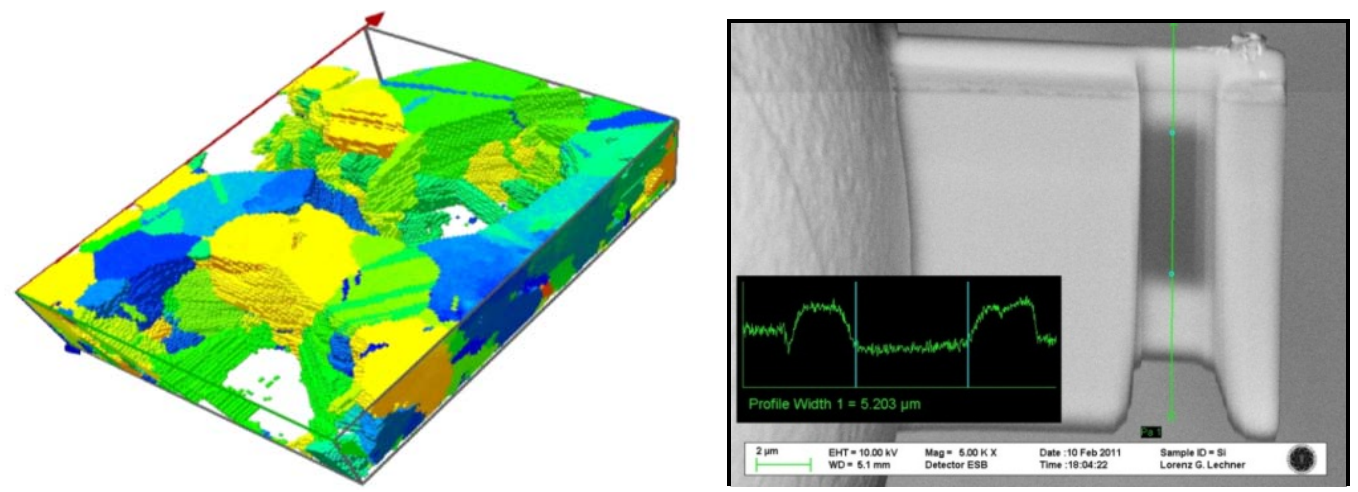

Figure 2. Crossbeam provides robust Solutions for 3D-Analytics (left) or Sample preparation (Right, Ultra thin TEM lamella)
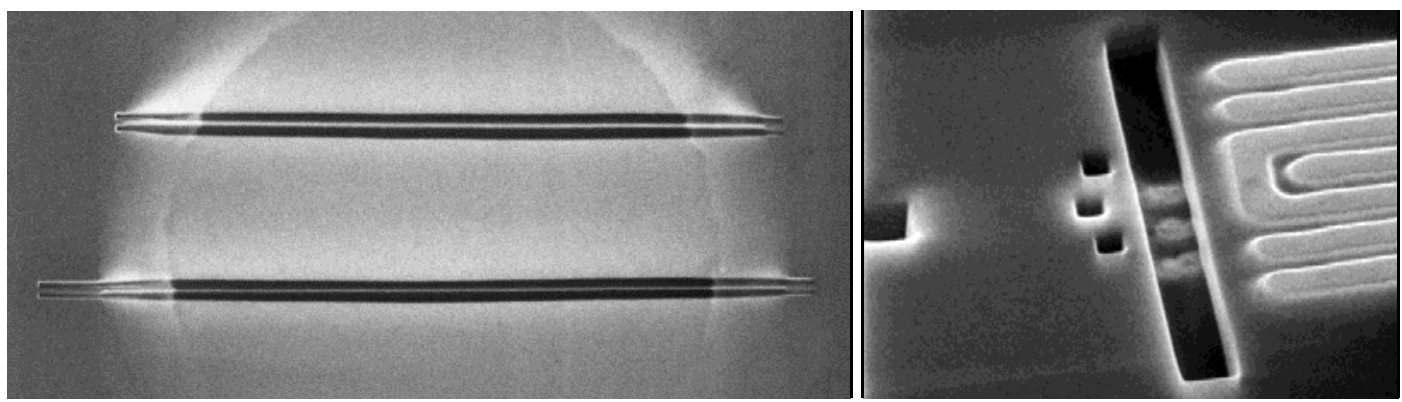

Figure 3. Sub-20 nm Graphene Nanoribbons (left) and edited $22 \mathrm{~nm}$ circuit (right) processed on the ORION Nanofab 